\title{
Noise Filtering Design of Non-intrusive Electrical Monitoring System
}

\author{
Xinghao $\mathrm{Wei}^{1, \mathrm{a}}$, Bo Yin ${ }^{2, \mathrm{~b}}$ and Zhicheng Zhu ${ }^{3, \mathrm{c}}$ \\ 1,2,3 Ocean University of China, No.238, Songling Road, Laoshan District, Qingdao City, \\ Shandong Province, China \\ a weixinghao_01@sina.com, ${ }^{b}$ ybfirst@163.com, ${ }^{\mathrm{c}}$ zhuzcwork@126.com
}

Keywords: Non-intrusive, noise filter, filter circuit, analog to digital conversion, Gabor filter

\begin{abstract}
With the electric energy consumption monitoring equipment is the foundation to carry out energy-saving work, strengthening the monitoring of energy consumption especially power consumption monitoring work in China to improve energy efficiency, realize the sustainable development of the energy, construction of a conservation oriented society and ease the pressure of energy is of great significance. This paper carried out a new electric power load energy consumption monitoring method non-intrusive power load research and monitoring of the decomposition, breaking the current status of power can only be carried out monitoring of total amount of power load, power monitoring, further refinement to the internal load of different electrical appliances, this paper presents a design method of non-intrusive noise filtering the type of power load decomposition. The method in the current signal from the circuit, firstly the signal of a series of filtering through the filter circuit is designed to filter the noise and interference, and then the signal of high precision analog to digital conversion, and then use Gabor filter re filtering operation, the signal is more stable and clean. In order to complete the non intrusive power detection system hardware design, to ensure the stability and integrity of current signal, and can be completed next to the relevant signal processing.
\end{abstract}

\section{Introduction}

Non-invasive monitoring of electric load of this technology, originally developed by Hart in 1980s when raised, it is in the development of monitoring tools a basic does not have what effect, to provide specific data for residential users of electrical equipment power consumption for electric power companies[1,7]. It is essentially different from intrusive load monitoring, intrusive load monitoring is a load installed in each load before the electricity detection device to measure the electrical characteristics of a load, this method is very complicated, and very high cost, not for all users, can not meet the electric power company the residents and the needs of users, and non intrusive load monitoring can easily solve these problems, just set up a non-intrusive load monitoring of electrical equipment in power supply switch at the entrance, according to the collected total power of signal precipitation single appliances electricity information[2-4]. This method can make the power company to get electricity consumption information, and can carry out the reasonable allocation of electricity, reduce energy waste, for users, can get the power consumption of each load their own family, in time of high power ageing equipment replacement, thereby reducing power consumption and unnecessary waste[5,8,9] .

Because of noise and interference in the circuit, there is a very serious effect on the electrical signal, we can make the signal for the noninvasive monitoring of electric load are more stable, the measured current signal is more clear, mentioned in this article, a design method for noise filtering of non intrusive load monitoring. The signal can be monitored, more conducive to the back related to signal processing, including event detection, feature extraction, cutting pattern classification,recognition and so on ${ }^{[6]}$. Signal processing is the key of this paper. In this paper, we describe in detail the process of getting the sampling signal, and can get the best stable waveform which is easy to handle and the noise is very little. 


\section{Design of Non-intrusive Electrical Load Monitoring System}

In the non-intrusive load monitoring system, data sampling design is the most important part of the bottom, it is related to the acquisition of the signal switching event monitoring, feature extraction and a series of operations, signal acquisition can make stable data more reliable and give the guarantee to complete the subsequent operation.

The Basic Block Diagram of Hardware Design.

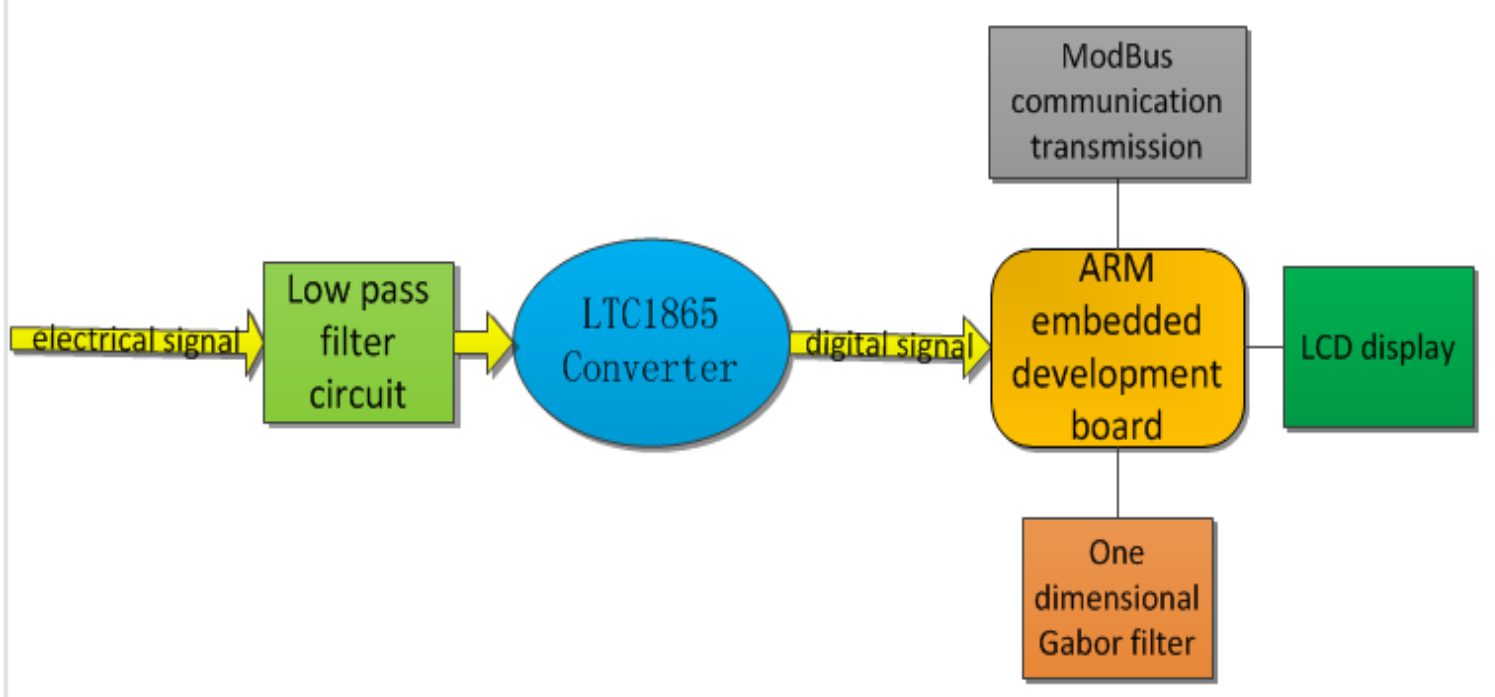

Fig 1.Hardware design block diagram of non-intrusive load monitoring system

Design of Low Pass Filter Circuit.In the block diagram, the design of the low pass filter circuit plays a key role in the hardware. The basic principle of the filter is to use the frequency characteristics of the circuit to realize the choice of the frequency components of the signal. According to the frequency filtering, the signal is regarded as the analog signal from the different frequency sine wave superposition, through the choice of different frequency components to achieve the signal filtering. Which allows the signal in the lower frequency components through the filter, which is called low pass filter, good low-pass filter design can make the circuit interference and noise is very good filter. This non-intrusive load monitoring system of low pass filter circuit design as shown below:

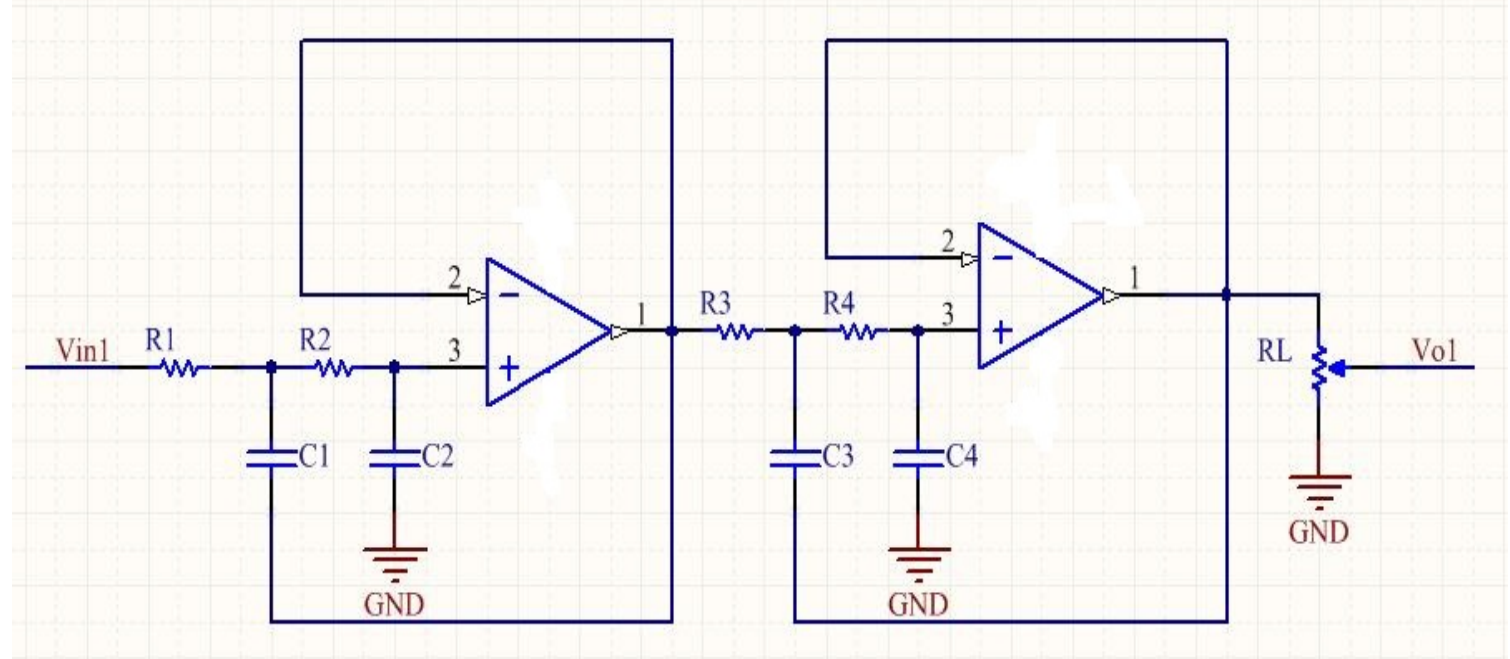

Fig 2.Low pass filter circuit design

Circuit Filtering Effect. Circuit filtering effect will be shown in the following figures: 


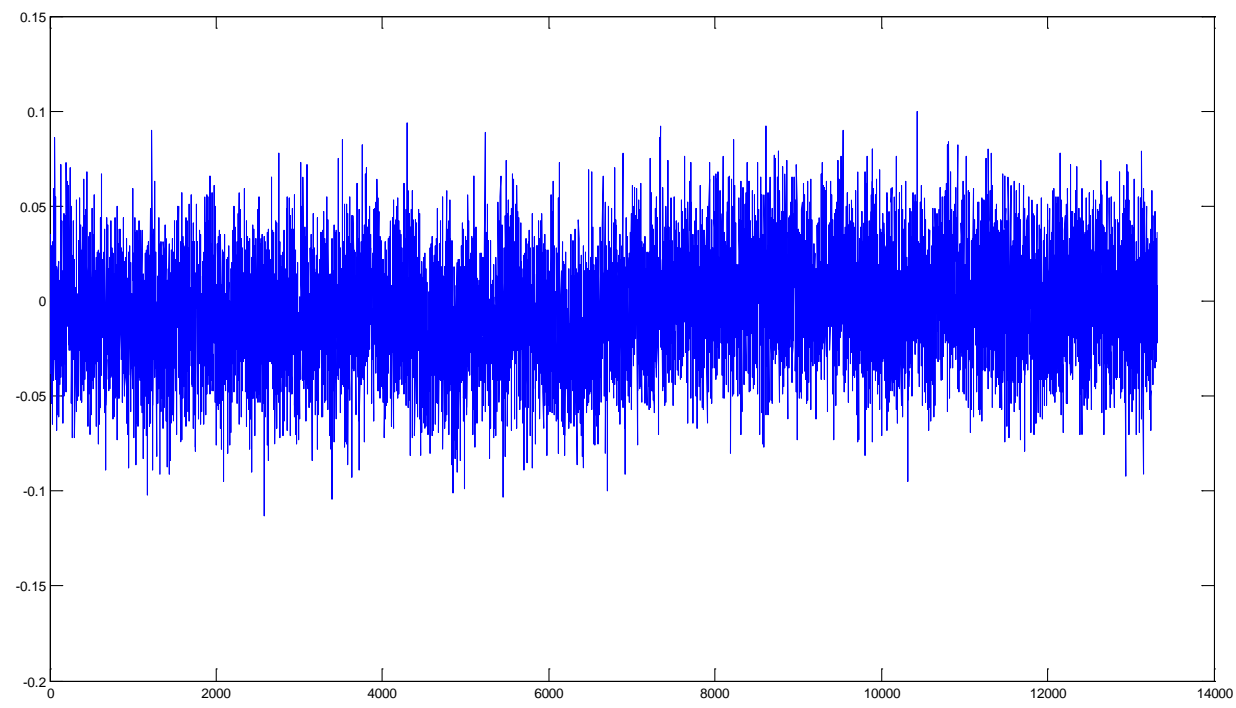

Fig 3. No-load current waveform before filtering

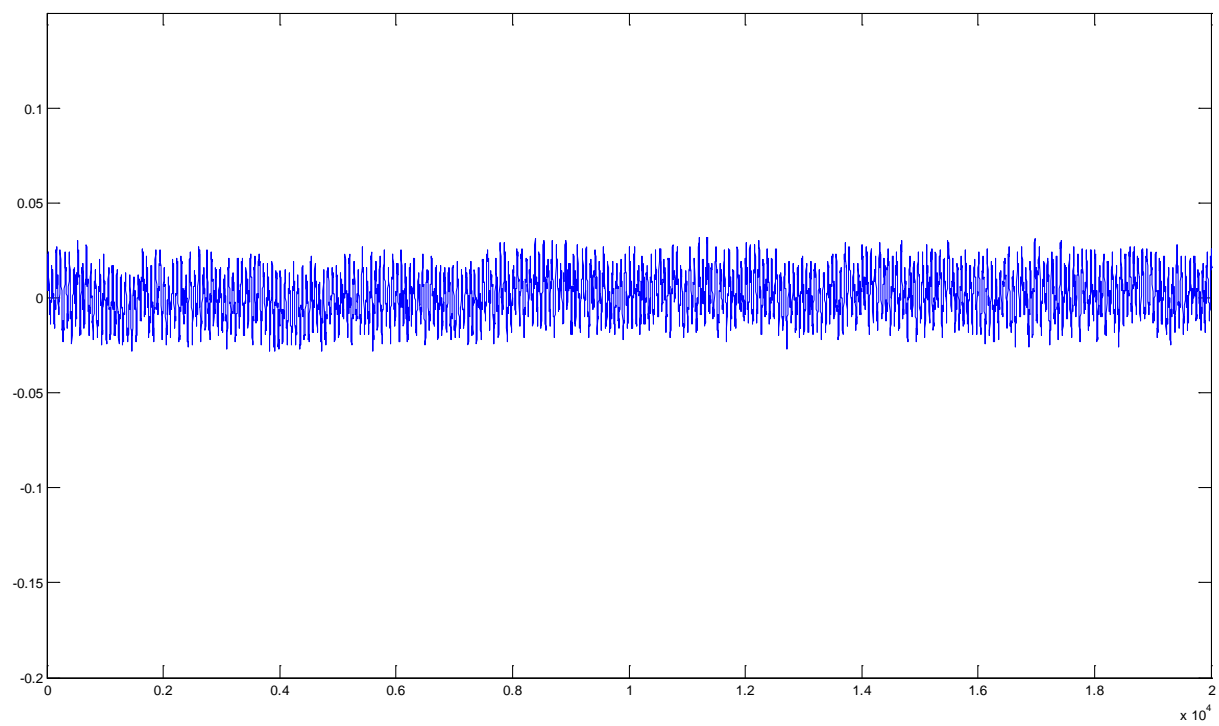

Fig 4. No-load current waveform after filtering

As can be seen from the above Fig 3 and Fig 4, after the circuit filter, the noise and interference in the circuit has been greatly improved, so that the current can be more stable and clear. For the above obtained image signal to noise ratio analysis, the signal to noise ratio before the filter is $56 \mathrm{~dB}$, after filtering the signal to noise ratio is $82 \mathrm{~dB}$.

\section{Electrical Signal Processing Based on One Dimensional Gabor Filter}

One Dimensional Gabor Filtering Algoritm.One dimensional Gabor filter is a band pass filter, which can be defined as a complex exponential function modulated by Gauss's function[10,11], $\mathrm{f}(\mathrm{t})$ is a one-dimensional current digital signal, whose Fourier transform is

$$
G(f, w)=\int_{-\infty}^{\infty} f(t) e^{-i w t} d t
$$

The obtained signal and Gauss's function

$$
\mathrm{g}_{\sigma}(\mathrm{t})=\frac{1}{\sqrt{2 \pi} \sigma} \exp \left(-\frac{\mathrm{t}^{\wedge} 2}{2 \sigma^{\wedge} 2}\right)
$$

convolution, The window can be added 


$$
G(f, \sigma, p, w)=\int_{-\infty}^{\infty} f(t) g_{\sigma}^{*}(t-p) e^{-i w t} d t
$$

This is a one-dimensional Gabor filtering principle, from the above equation can be seen, when $t$ get to $p$, Gauss function to the maximum, $t$ extends to both sides, then $f(t)$ value was rapidly weakened, the signal changes of Gabor at $\mathrm{P}$ point is mainly the $3^{*} \sigma$ range of $\mathrm{P}$ point, and far more small far, $\mathrm{P}$ will turn to move in the timeline, you can get signal analysis in time domain, so that the current signal filtering in the frequency domain, the denoising effect can better improve.

Experimental Results of Gabor Filter. Experimental results of Gabor filter will be shown in the following figures:

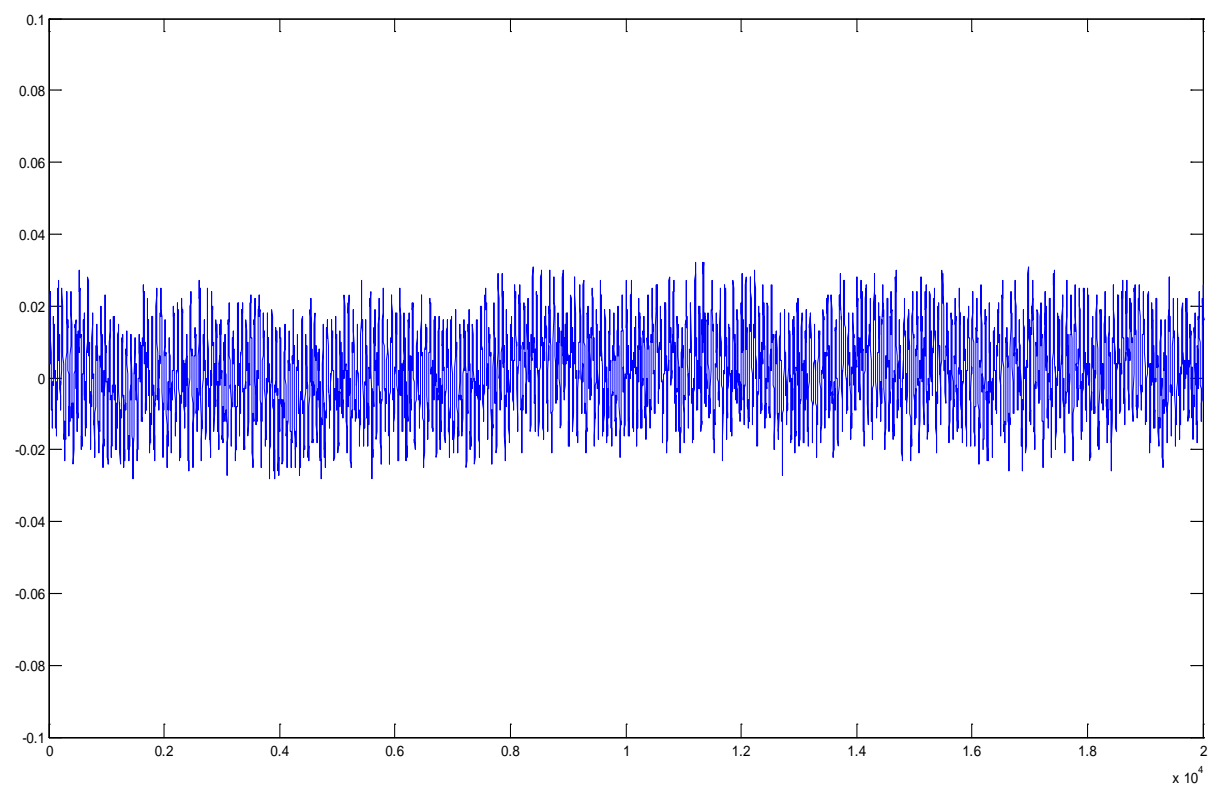

Fig 5.No-load current waveform before Gabor filtering

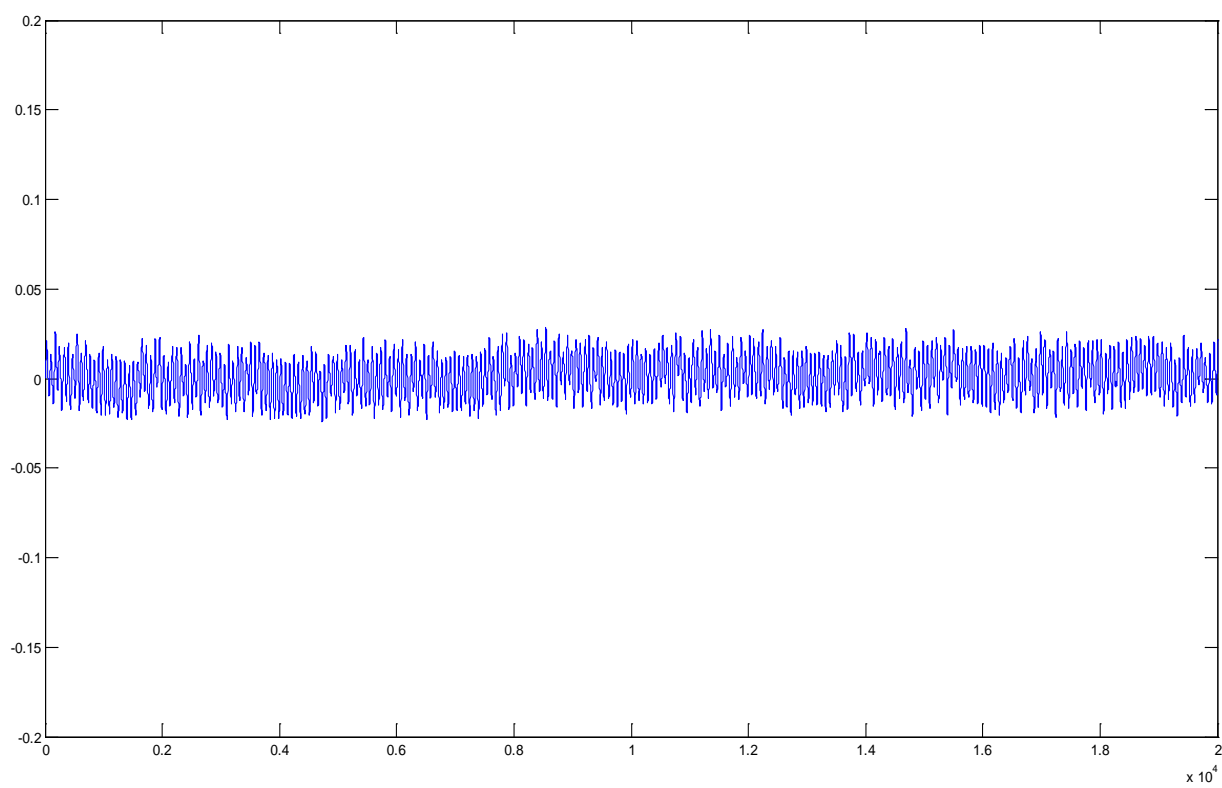

Fig 6.No-load current waveform after Gabor filtering

From Fig 5 and Fig 6 can be seen after the one-dimensional Gabor filter effect, in the case of noload circuit, after Gabor filtering after the signal stability has been significantly improved, a lot of noise are filtered, the signal characteristics can be well reflected from the experimental results to the analysis, we can see have the relevant load. 


\section{Analysis of Experimental Results}

We grew up on power to many different load power test data acquisition design, respectively, fan, lamp, electric kettle, electric heating, oxygen machine etc.. Through the MATLAB tracing of current data obtained from the real situation, observe the depicted figures are the same and all kinds of electrical work, in order to determine the reliability and practicability of the non intrusive load monitoring system filtering method. The current digital signal collected by the electric lamp and electric kettle is drawn in MATLAB as shown in Fig 7 and Fig 8.

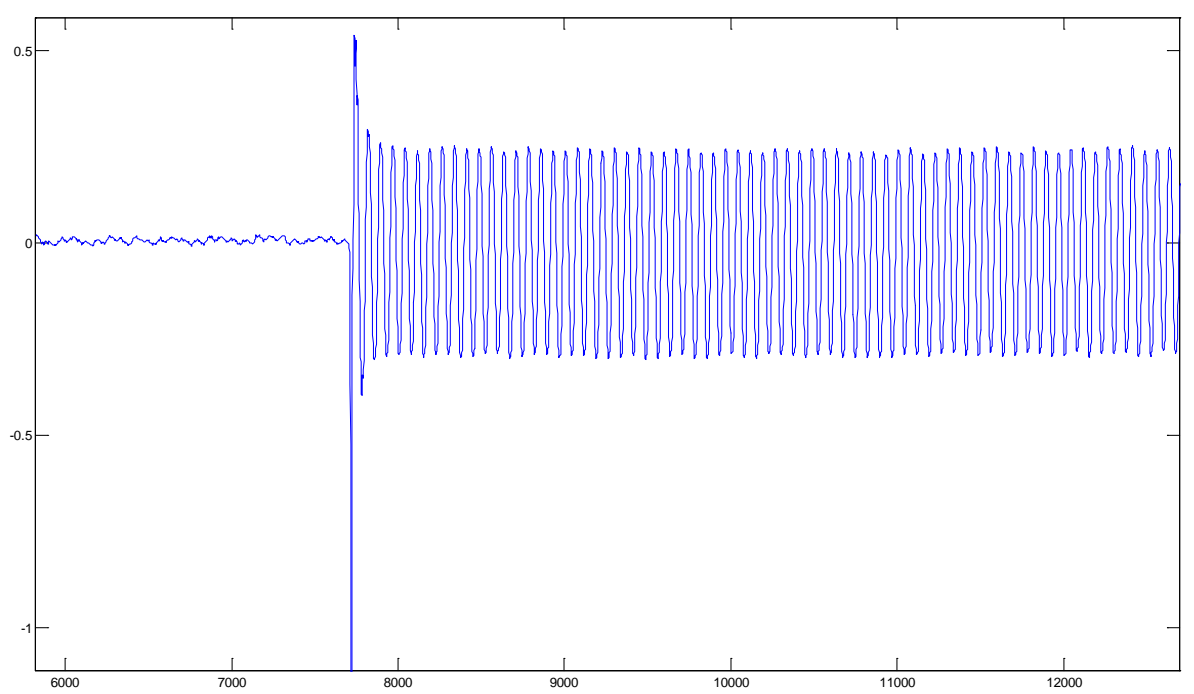

Fig 7. The electric current of the electric light is drawn in MATLAB, and the horizontal coordinate is the sequence of data points and the current $(A)$.

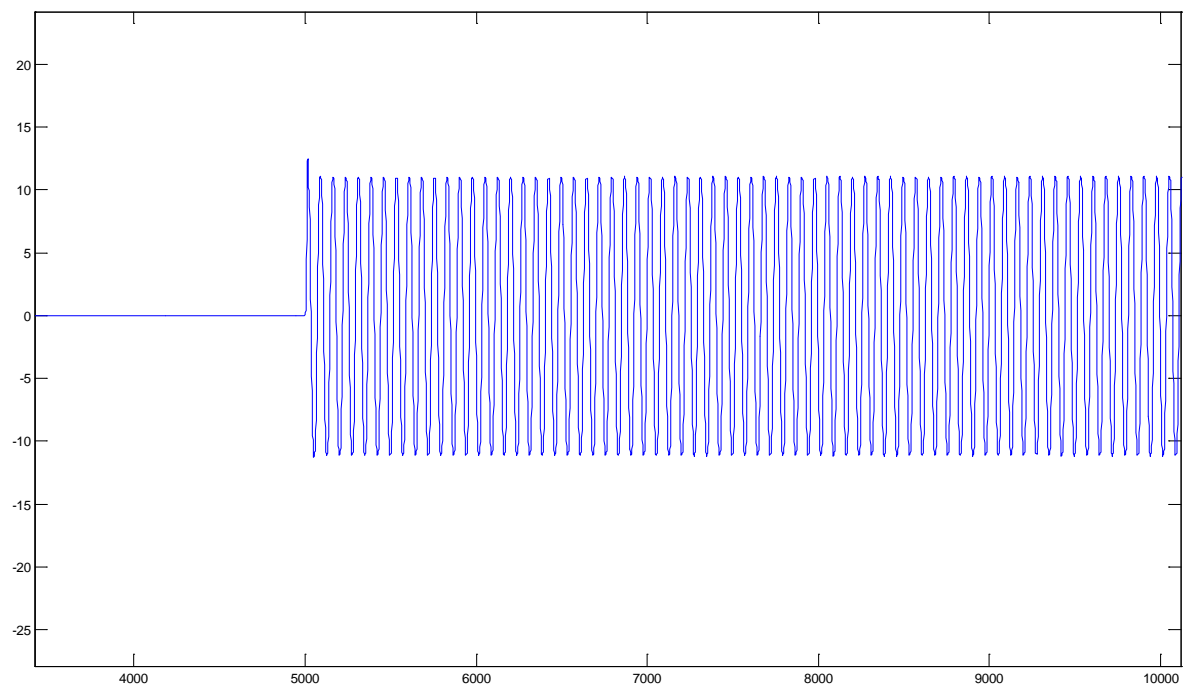

Fig 8.Electric kettle of the current in the MATLAB drawing, the horizontal coordinates of the data point sequence, the vertical axis of the current (A).

From the above two picture can be seen in the light and electric kettle by opening the event process data is very stable and reliable, the design method of the filter we completely for non intrusive load monitoring system, to the maximum extent reflect the current in circuit. 


\section{Conclusion}

Non-intrusive electrical monitoring system can be better on the ground to the user, and become the main development trend of the future load monitoring, the researchers are actively carry out research work[12]. The design method of data acquisition for this system can provide a good platform for filter design, through the analysis of the above experimental results, we can see that the data acquisition device designed by us can well reflect the real situation of all kinds of electrical appliances in the practical work, to ensure that the current data accuracy and stability lay a good foundation for the follow-up so as to extract the current signal processing, event monitoring and switching characteristics.

In addition, the related design non intrusive load monitoring can save cost, reduce unnecessary waste of hardware costs are relatively high, economy and applicability, the implementation is very high, can well satisfy the non intrusive load monitoring system requirements.

\section{Acknowledgements}

This work was financially supported by International S\&T Cooperation Program of China (2015DFR10490).

\section{Reference}

[1] Hart G W.Nonintrusive appliance load monitoring[J]. Proceedings of the IEEE,1992,80(12):1870-1891

[2] ERGEG [European Regulators' Group for Electricity and Gas]. Position paper on smart grids. An ERGEG public consultation paper. Ref: E09-EQS-30-04; 10 December, 2009.

[3] Wissner M. The smart grid - A saucerful of secrets?. Appl Energy 2011;88:2509-18.

[4] Nagesh DYR, Krishna JVV, Tulasiram SS. A real-time architecture for smart energy management. Innovative Smart Grid Technol 2010:1-4.

[5] DECC [Department of Energy and Climate Change]. Impact assessment of a Gbwide smart meter rollout for the domestic sector, London; 2009.

[6] L.I. Eguiluz, M. Manana, J.C. Lavandero, Voltage distortion influence on current signatures in nonlinear loads, in: IEEE Power Engineering Society Summer Meeting, vol. 2, 2010, pp. 11651170.

[7] Y.H. Lin, M.S. Tsai, C.S. Chen, Applications of fuzzy classification with fuzzy Cmeans clustering and optimization strategies for load identification in NILM systems, in: IEEE Int. Conf. on Fuzzy Systems, 2011.

[8] J.S.K. Leung, K.S.H. Ng, J.W.M. Cheng, Identifying appliances using load signatures and genetic algorithms, in: Int. Conf. of Electrical Engineering, July, 2007.

[9] M. Dong, P.C.M. Meira, W. Xu, C.Y. Chung, Non-intrusive signature extraction for major residential loads, IEEE Trans. Smart Grid 4 (2013) 1421-1430.

[10] Lee T S. Image representation using 2D Gabor wavelets[J]. IEEE Trans on Pattern Analysis and Machine Intelligence, 1996, 18(10): 959-971.

[11] TSAID M, WU S K. Automated surface inspection using Gabor filters[J]. International Journal of Advanced Manufacturing Technology,2000,16(7):474-482.

[12] L. Jiang, S. Luo, J. Li, An approach of household power appliance monitoring based on machine learning, in: 5th International Conference on Intelligent Comuputation Technology and Automation, 2012, pp. 577-580. 